\title{
Uma Proposta de Incubadora Multissensorial para Neonatos: Das Práticas Médicas com o Robô Premature Anne às Possibilidades de Transformação em Produto de Baixo Custo
}

\author{
Letícia de Almeida Reche ${ }^{1}$, João Benedito dos Santos Junior ${ }^{2}$, Júlia Pereira \\ Soares $^{3}$, Norma Ávila da Silveira ${ }^{3}$, Núbia Samara Maia ${ }^{3}$, Thatia Regina Bonfim ${ }^{4}$, \\ Mayra Loures de Oliveira ${ }^{5}$
}

\footnotetext{
${ }^{1}$ Aluna de Graduação do Curso de Ciência da Computação da PUC Minas Campus de Poços de Caldas - MG, Brasil

${ }^{2}$ Orientador e Docente do Departamento de Ciência da Computação e do Departamento de Medicina da PUC Minas Campus de Poços de Caldas - MG, Brasil

${ }^{3}$ Alunas de Graduação do Curso de Medicina da PUC Minas Campus de Poços de Caldas - MG, Brasil

${ }^{4}$ Co-orientadora e Docente do Departamento de Medicina e do Departamento de Fisioterapia da PUC Minas Campus de Poços de Caldas - MG, Brasil

${ }^{5}$ Aluna de Graduação do Curso de Medicina do Centro Universitário de Caratinga - MG, Brasil

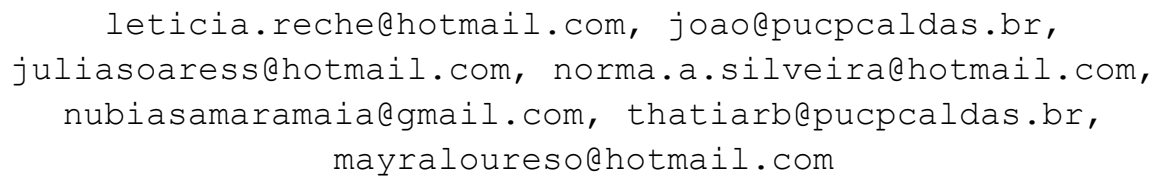

\begin{abstract}
This work presents a prototype proposal for a Multisensory Neonatal Incubator, for academic purposes and use in a hospital environment. In the case of use in academic environments, it is intended to improve the quality of the training environment of medical students and other courses in the area of Health, offering a very realistic environment, when compared to the environment that many doctors find in everyday situations of care with newborns. In the case of use in hospital environments, it is intended to construct a low-cost but efficient and reliable equipment that can be applied to reduce the important deficit of incubators for newborns existing in Brazil. The prototype is developed in a multidisciplinary way, with students and teachers from the both Medicine and Computer Science courses, taking advantage of the resources and simulation features offered by the Premature Anne robot-mannequin.
\end{abstract}

Resumo. Este trabalho apresenta uma proposta de protótipo de Incubadora Neonatal Multissensorial, para fins acadêmicos e eventual uso em ambiente hospitalar. No caso de uso em ambientes acadêmicos, pretende-se melhorar a qualidade do aprendizado de estudantes de Medicina e demais cursos da área de Saúde, oferecendo um ambiente bastante realístico, quando comparado ao ambiente que muitos médicos encontram nas situações cotidianas de atendimento a recém-nascidos. No caso de uso em ambientes hospitalares, pretende-se chegar à construção de um equipamento de baixo custo, porém eficiente e confiável, que possa ser aplicado para reduzir o déficit de incubadoras para recém-nascidos existente no Brasil. O protótipo é desenvolvido de forma multidisciplinar, com alunos e professores dos cursos de Medicina e Ciência da Computação, usufruindo dos recursos e funcionalidades de simulação oferecidos pelo robô-manequim Premature Anne. 


\section{INTRODUÇÃO}

No Brasil, as mortes entre recém-nascidos representam, aproximadamente, $70 \%$ do total de óbitos registrados no primeiro ano de vida. Além disso, em todo o território nacional, $11,9 \%$ das crianças nascem prematuras, segundo dados do Sistema Nacional de Nascidos Vivos, do Fundo das Nações Unidas para a Infância (UNICEF). Observa-se, então, que se trata de uma taxa extremamente alta, se comparada àquelas registradas em outros países com características similares ao Brasil. De forma complementar, as taxas de recém-nascidos, com baixo peso ao nascer, têm permanecido em torno de 8\% [1][2].

Em linhas gerais, entre as principais causas dos óbitos neonatais, estão problemas respiratórios, asfixia ao nascer, infecções, distúrbios metabólicos, dificuldade para se alimentar e dificuldade para regular a temperatura corporal [3]. Sabe-se, ainda, que a hipotermia está fortemente associada ao aumento da mortalidade e morbidade neonatais, sendo motivo de preocupação, uma vez que os recém-nascidos acometidos por essa situação podem desenvolver diversos tipos de doenças e até evoluir para óbito [4]. Nesse sentido, a permanência do recém-nascido de alto risco nas Unidades de Terapia Intensiva Neonatais (UTIN) torna-se necessária, e o uso da Incubadora Neonatal é de extrema importância, pois contribui para a prevenção de muitas intercorrências, se ambos estiverem em condições físicas adequadas [5][6].

\section{EVOLUÇÃO DAS INCUBADORAS PARA RECÉM-NASCIDOS}

As primeiras incubadoras chegaram ao Brasil no início do século XX e sempre tiveram como principal incumbência evitar a hipotermia e, consequentemente, aumentar a sobrevida do recém-nascido. Durante muitos anos, foram empregados recursos para manter a temperatura corporal do prematuro com meios improvisados, que podiam causar danos à integridade da pele dos recém-nascidos [8]. No entanto, os avanços da ciência, das práticas da neonatologia e das tecnologias permitiram que as incubadoras se tornassem um ambiente mais adequado para o prematuro. Assim, ao longo do tempo, a principal função das incubadoras para recém-nascidos foi permitir que o bebê mantivesse uma temperatura ideal corporal e constante, embora também permitisse regular a umidade do ar. Em termos regulatórios, ainda que a maioria dos prematuros permaneça por várias semanas na incubadora, deve-se considerar a meta de permanência até que o seu peso atinja, no mínimo, os $2 \mathrm{Kg}$ [3].

\section{PROBLEMAS ESTRUTURAIS DAS INCUBADORAS}

Embora a Incubadora Neonatal seja considerada como um equipamento que revolucionou a Medicina ao longo de várias gerações, deve-se que observar que muitos destes equipamentos, no contexto de uma UTIN, não funcionem adequadamente, seja por falta de recursos tecnológicos, seja por falta de profissionais de saúde com as devidas habilidades e treinamento. Assim, ao invés de oferecerem suporte ao tratamento das necessidades do recém-nascido, podem representar riscos à vida dos mesmos. Esses riscos estão relacionados a três fatores essenciais: a) ao número insuficiente de incubadoras, devido aos custos financeiros para sua aquisição, conforme pode ser visto na Tabela 01; b) aos níveis de ruído que muitos equipamentos produzem durante sua operação, não oferecendo mecanismos tecnológicos sensoriais para o monitoramento efetivo das condições ambientais dentro e fora da incubadora; c) à inabilidade de muitos profissionais da área da saúde na operação desses equipamentos, tanto pela falta de 
contato com os mesmos quanto pelo nível insuficiente de treinamento para sua operação.

Tabela 01 - Exemplos de Incubadoras para Neonatos e seus Custos

\begin{tabular}{|c|c|l|}
\hline MODELO & VALOR & \multicolumn{1}{|c|}{ PRINCIPAIS CARACTERÍSTICAS } \\
\hline $\begin{array}{c}\text { Incubadora neonatal } \\
\text { 1186A FANEM }\end{array}$ & $\begin{array}{c}\text { Aproximadamente } \\
\mathrm{R} \$ 22.000\end{array}$ & $\begin{array}{l}\text { Aquecimento: Modo Pele e Modo Ar; Operação simples; } \\
\text { Oxigênio Servocontrolado (versão C); Umidade Controlada } \\
\text { (versão C) e Umidade passiva (versão A); Oxímetro de } \\
\text { pulso integrado SpO2 (versão C). }\end{array}$ \\
\hline $\begin{array}{c}\text { Incubadora neonatal } \\
\text { 1186c FANEM }\end{array}$ & $\begin{array}{c}\text { Aproximadamente } \\
\text { R } \$ 25.000\end{array}$ & $\begin{array}{l}\text { Painel de LCD monocromático com 5,5" com exibição de } \\
\text { parâmetros gráficos; Aquecimento: Modo Pele e Modo Ar; } \\
\text { Operação simples; Leito deslizante, radio-transparente e } \\
\text { com sistema de inclinação para facilitar o atendimento do } \\
\text { paciente; Sistema de Umidificação Servo-controlada; Sensor } \\
\text { de temperatura auxiliar. }\end{array}$ \\
\hline $\begin{array}{c}\text { Incubadora neonatal } \\
\text { MILLENNIUM com } \\
\text { acessórios - Gigante }\end{array}$ & Aproximadamente & $\begin{array}{l}\text { Indicação visual e sonora dos alarmes de Hipotermia, } \\
\text { hipertermia, subtemperatura, sobretemperatura, falha no } \\
\text { sistema, falha de circulação de Ar na cúpula, falha de } \\
\text { aquecimento, falta de energia, sensor RN desconectado, } \\
\text { falha no sensor e cúpula aberta. }\end{array}$ \\
\hline $\begin{array}{l}\text { Incubadora neonatal } \\
\text { estacionária OLIDEF }\end{array}$ & Aproximadamente \\
modelo LINE 4 & R $\$ 37.000$ & $\begin{array}{l}\text { Display alfanumérico com indicação simultânea das } \\
\text { temperaturas desejada, do Ar, do paciente, peso e umidade } \\
\text { relativa do ar; Certificado de acordo com as normas: NBR } \\
\text { IEC 60601-1, NBR 60601-2-19, NBR 60601-1-2 e NBR } \\
\text { 60601-2-49. Ampla linha de opcionais }\end{array}$ \\
\hline
\end{tabular}

\section{PROPOSTA DE UM PROTÓTIPO MULTISSENSORIAL E INTELIGENTE}

Conforme citado, este trabalho tem como objetivo apresentar a criação de um protótipo de Incubadora Neonatal Multissensorial e minimente Inteligente (smart, do ponto de vista de favorecer reações automáticas, controladas e confiáveis, com o mínimo de intervenção humana), tanto para fins acadêmicos quanto para o uso em ambiente hospitalar. Do ponto de vista acadêmico, pretende-se utilizar a incubadora para favorecer a formação e o treinamento de estudantes de Medicina e/ou de cursos da área da Saúde. Do ponto de vista das unidades hospitalares, pretende-se oferecer uma plataforma de hardware e software, configurando-se um ambiente de computação embarcada, com aspectos de alta tecnologia, porém de menor custo, quando comparada a alguns equipamentos disponíveis no mercado, o que pode viabilizar o seu uso em maior escala, beneficiando, principalmente, localidades onde esse tipo de equipamento é escasso e/ou inexiste.

Desde a fase de especificação, o projeto da Incubadora Multissensorial e Inteligente para Recém-Nascidos tem sido desenvolvido de forma interdisciplinar e multidisciplinar, envolvendo conhecimentos advindos do curso de Medicina - que contribui com a especificação dos parâmetros ideais para o funcionamento da incubadora, do ponto de vista da Neonatologia - e com conhecimentos advindos da área da Ciência da Computação - que contribui com as questões de implementação, validação e testes do ambiente tecnológico proposto.

\subsection{Metodologia de Pesquisa}

Ao longo das fases de desenvolvimento da incubadora, atividades contínuas de pesquisa em bancos de dados, tais como Medline e Scielo, e em cadernos do Ministério da Saúde, foram muito importantes para identificação dos principais aspectos relacionados aos 
problemas nas incubadoras e como eles poderiam ser evitados. Estudos e pesquisas de campo em uma UTIN também foram realizados, além de entrevistas técnicas com profissionais que trabalham com equipamentos dessa natureza. Aspectos da Medicina Baseada em Evidências, desenvolvidos no contexto em que este trabalho se insere, foram considerados e contribuem de forma expressiva com os resultados preliminares obtidos [7].

Construído o protótipo de hardware e software da incubadora, foram realizadas configurações específicas no robô-manequim Premature Anne, produzido pela empresa norueguesa Laerdal e disponível para simulações no ambiente dos Laboratórios de Anatomina do curso de Medicina. O robô-manequim Premature Anne, quando em estado de operação, oferece um conjunto avançado de parâmetros para monitoramento, se aproximando muito do estado corpóreo de um recém-nascido. A figura 1 apresenta o robô-manequim Premature Anne.

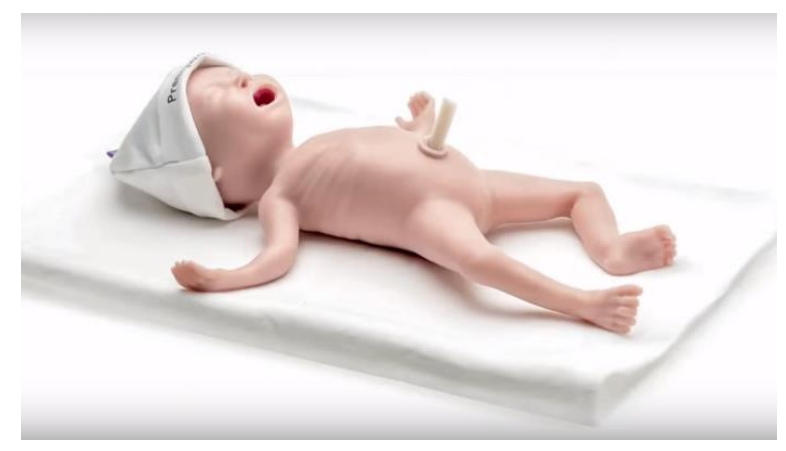

Figura 1: robô-manequim Premature Anne

Do ponto de vista do hardware, a Incubadora Multissensorial e Inteligente para RecémNascidos é um projeto baseado em sensores, sistemas embarcados de controle (como é o caso do Arduíno e Raspebery) e câmeras para monitoramento a distância (que a inserem também num contexto de Telemedicina).

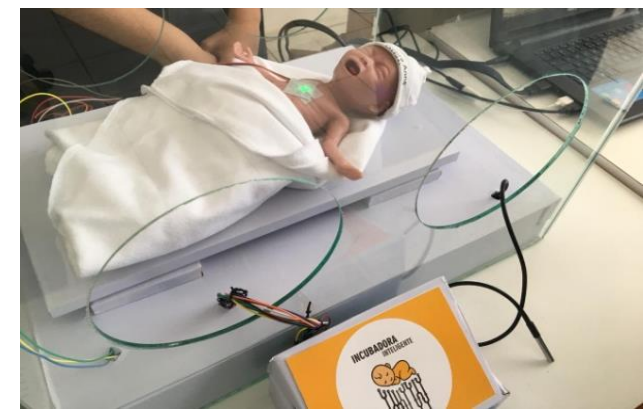

Figura 2a: Estrutura interna

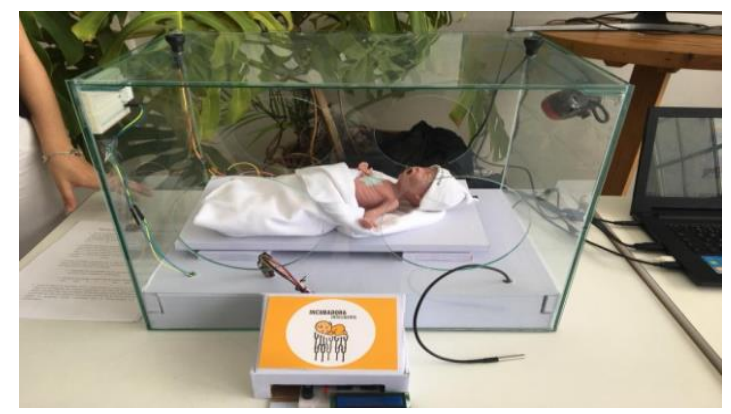

Figura 2b: Controlador de sensores

No caso do conjunto de sensores, a plataforma é composta por sensor de frequência cardíaca, temperatura corporal, temperatura e umidade do ambiente (para realizar a medição dentro da incubadora), sensor de presença de gases tóxicos (como o monóxido de carbono e a amônia), além de uma célula de carga, capaz de realizar a medição do peso corporal. As figuras $\mathbf{2 a}$ e $\mathbf{2 b}$ apresentam a estrutura da incubadora. 


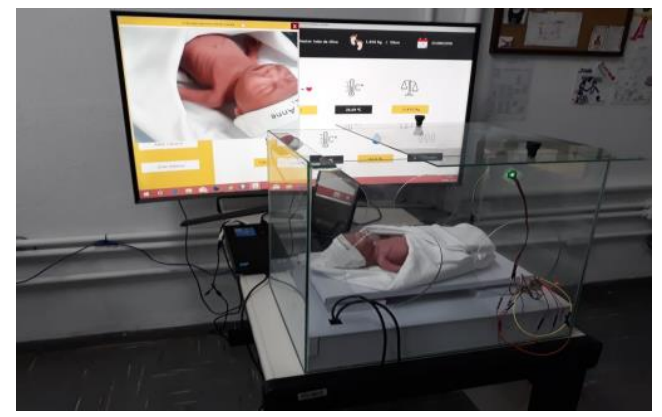

Figura 3: Ambiente completo proposto para a incubadora

Do ponto de vista do software, ferramentas, API (Application Programming Interfaces) e pacotes de desenvolvimento Java foram utilizados na implementação. Em relação às aplicações em Telemedicina, a Incubadora conta com câmeras instaladas no seu interior, possibilitando a visualização e acompanhamento do recém-nascido em tempo real e em localidades distantes. A plataforma de software recebe dados dos sensores em tempo real, processa esses dados e produz informações valiosas, que podem ser visualizadas em múltiplas telas (notebooks, televisores, tablets e smartphones). A figura 3 apresenta o ambiente integrado de hardware e software proposto para uso da incubadora.

\subsection{Ambiente e Cenários de Testes}

Para validar o uso da incubadora, cenários de simulação clínica foram configurados e construídos usando o robô-manequim Premature Anne; esses cenários são construídos com o uso de um tablet, que acompanha o equipamento robótico, sendo utilizada a interface de rede bluetooth para transferência de dados. Para a construção de um cenário, são definidos parâmetros para os sinais vitais, e o robô passa a reagir de acordo com os mesmos. Então, os sensores da incubadora capturam os dados de monitoramento, o que permite a simulação de um ambiente próximo de um ambiente real. Uma vez obtidos os dados, o protótipo da incubadora é capaz de tomar decisões, de maneira automática e/ou controlada, sobre as melhores combinações de parâmetros para que o ambiente da incubadora se ajuste às demandas do corpo do bebê prematuro. Assim, além de informar, em tempo real, os sinais vitais do recém-nascido, a plataforma emite notificações e alertas visuais, permitindo ações mais rápidas dos profissionais envolvidos no cuidado do prematuro. As notifiçações de alerta são emitidas para o ambiente local, no qual a incubadora está inserida, bem como para acompanhamento remoto, de acordo com os registros de dados dos profissionais habilitados e autorizados a receber tais notificações.

\section{RESULTADOS PRELIMINARES E CONTRIBUIÇÕES}

A partir da análise dos dados obtidos com o teste da Incubadora Inteligente usando o robô-manequim Premature Anne, e confrontando os resultados de simulações com a realidade das UTINs do país, pôde-se observar o potencial para estudos avançados em pesquisa e as possibilidades para o desenvolvimento de um equipamento para uso hospitalar [8]. Os principais problemas citados ao longo deste artigo e as intercorrências que podem ser evitadas durante a operação de equipamentos dessa natureza constituem as principais motivações para a sequência deste trabalho, que, no contexto de pesquisa da Pontifícia Universidade Católica de Minas Gerais, possui investimentos para seu 
desenvolvimento até o final de 2021. De forma complementar, por simular condições próximas de um ambiente real, o protótipo da incubadora pode evoluir para uso em ambiente acadêmico e para um produto de baixo custo, uma vez que o preço dessas incubadoras neonatais, conforme mostrado na Tabela 01, é um fator que impacta o seu uso no Brasil. Estudos de viabilidade iniciais indicam que é possível projetar e construir a incubadora, proposta neste trabalho, com custo aproximado de dez mil reais.

\section{CONSIDERAÇÕES FINAIS}

Ao longo do desenvolvimento deste trabalho, que está entrando em sua segunda etapa de desenvolvimento, ao longo de 2019, observou-se que o problema das incubadoras neonatais é real e relativamente grave, principalmente pela insuficiência de equipamentos, em face à demanda. De acordo com o artigo IV da Declaração Universal dos Direitos do Bebê Prematuro, todos os prematuros possuem direito ao tratamento estabelecido pela ciência, sem distinções, portanto, todos devem receber tratamentos e chances iguais de sobrevivência e desenvolvimento [9]. Em linhas gerais, pretende-se, conforme citado, viabilizar um produto acessível a todas as Unidades de Terapia Intensiva Neonatal em âmbito nacional, de modo a proporcionar um ambiente mais seguro possível aos recém-nascidos.

\section{REFERÊNCIAS}

[1] Barros, F; Matijasevich, A.; Silveira, M.: Pesquisa para estimar a prevalência de nascimentos pré-termo no Brasil e explorar possíveis causas. Consultoria UNICEF Brasil. Julho, 2013.

[2] Portal ODM [homepage na Internet]. Brasil reduz mortalidade infantil em 83\%. Disponível em: http://www.portalodm.com.br/video/58/brasil-reduz-mortalidade-infantil-em-83. Último acesso em 01/03/2019.

[3] Tamez, R. N.; Silva, M. J. P.: Enfermagem na UTI neonatal: assistência ao recém-nascido de alto risco. $4^{\mathrm{a}}$ ed. Rio de Janeiro: Guanabara Koogan; (2009).

[4] Brasil. Ministério da Saúde. Secretaria de Atenção à Saúde. Departamento de Ações Programáticas e Estratégicas. Atenção à saúde do recém-nascido: guia para os profissionais de saúde / Ministério da Saúde, Secretaria de Atenção à Saúde, Departamento de Ações Programáticas e Estratégicas. Brasília: Ministério da Saúde, (2011).

[5] Costa, C. C.: Avaliação de incubadoras neonatais e práticas de manuseio pela equipe de enfermagem. Dissertação (Mestrado) - Faculdade de Medicina de Botucatu, Universidade Estadual Paulista Julio de Mesquita Filho, Botucatu, (2016).

[6] Costa, C. C.; Tonete, V. L. P.; Parada, C. M. G. L.: Conhecimentos e práticas de manuseio de incubadoras neonatais por profissionais de enfermagem. Acta paul. enferm. (2017); 30(2):174-80.

[7] OLIVEIRA, S.; VIEIRA, G. B.; SANTOS JUNIOR, J. B.; ALBANO, L.; JUNIOR, W. F.; Abrão, I. C.; BONFIM, T. R.: Tecnologias de Informação e a Medicina Baseada em Evidências: Possíveis Diálogos e Situações Controversas. In: XVI Congresso Brasileiro de Informática em Saúde, 2018, Fortaleza - CE. Anais do CBIS2018. São Paulo: SBIS - Sociedade Brasileira de Informática em Saúde, 2018. v.1.

[8] Soares, J.P.; Silveira, N. A.; Maia, N.S.; Oliveira, M. L.; Almedia, L. R.; Santos Junior, J. B. dos; Bonfim, T. R.: Uso de uma Incubadora Multissensorial para Práticas Médicas com Neonatos Usando o Robô Premature Anne. XV Congresso Paulista de Pediatria. São Paulo, março de 2019.

[9] Tavares, L. A. M.: Uma Declaração Universal de Direitos para o Bebê Prematuro. Brasil Diagraphic, (2008). Disponível em http://creativecommons.org/licencas/dominiopublico. Acesso em 30/01/2019. 